\title{
SYNTAX-Score II zur Therapiewahl bei komplexen Koronarerkrankungen?
}

\author{
Der SYNTAX-Score wird in den europäischen und \\ amerikanischen Leitlinien als Kriterium für die Therapiewahl \\ bei Patienten mit komplexen Koronarbefunden empfohlen. \\ Die Erweiterung der anatomischen Kategorisierung um \\ klinische Merkmale (SYNTAX-Score II) soll die prognostische \\ Relevanz erhöhen. Dies prüften nun Farooq et al. \\ Lancet 2013; 381: 639-650
}

Die SYNTAX-Studie war eine randomisierte, prospektive, multizentrische Studie, an der 1800 Patienten mit einer koronaren Dreigefäßerkrankung oder einer Stenose des linken Hauptstamms teilnahmen. Diese wurden randomisiert zu einer koronaren Bypassoperation oder zu einer perkutane Koronarintervention (PCI) mit einem Paclitaxel-freisetzenden Stent der 1. Generation. In die SYNTAX-Bewertung flossen Informationen zur physiologischen Bedeutung des stenosierten Gefäßes, Charakteristika der Läsion und eventuell vorhandene Bifurkationsläsionen ein. Bei Patienten mit einer Hauptstammstenose der linken Koronararterie waren eine perkutane Koronarintervention und eine Bypassoperation gleichwertig, wenn der SYNTAX-Punktwert $<33$ war. Patienten mit einer Dreigefäßerkrankung hatten bei Ergebnissen $<23$ eine vergleichbare Mortalität. Individualisierte Denkansätze und klinische Variablen wurden im Score bislang nicht erfasst. Farooq et al. ermittelten nun die Assoziationen zwischen der 4-Jahres-Mortalität und individuellen Charakteristika für Patienten mit Bypassoperation bzw. PCI.

Die beiden Patientengruppen waren hinsichtlich klinischer und soziodemographischer Daten vergleichbar. Prognostisch relevante Kriterien für den SYNTAX-

Score II waren

- der anatomische SYNTAX-Score,

- das Lebensalter,

- weibliches Geschlecht,

- die Kreatinin-Clearance,

- die linksventrikuläre Auswurffraktion (LVEF),

- eine Hauptstammstenose,

- periphere arterielle Verschlusskrankheiten und

- chronisch obstruktive Lungenerkrankungen.
Der SYNTAX-Score II prognostizierte signifikant einen Unterschied in der 4-Jahres-Mortalität zwischen den beiden Patientengruppen ( $\mathrm{P}_{\text {Interaktion }}=0,0037$ ). Die Kreatinin-Clearance und periphere arterielle Verschlusserkrankungen waren wenig bedeutsam, während die übrigen Kriterien eine mäßige bis starke Interaktion aufwiesen. Ein Diabetes mellitus war kein unabhängiger Prädiktor für die langfristige Mortalität ( $p=0,67$ ). Die Einbeziehung der relevanten klinischen Faktoren veränderte die Punktschwelle im SYNTAX-Score, bei dem die operative und perkutane Intervention die gleiche 4-Jahres-Mortalität hatten. Dies galt im unteren, mittleren und Hochrisikobereich. Patienten in jüngerem Alter, Patienten mit einer eingeschränkten LVEF und Frauen mussten für die gleiche Überlebenswahrscheinlichkeit einen geringeren Punktwert aufweisen. Bei älteren Patienten, Patienten mit COPD und solchen mit einer Hauptstammstenose war dies bei höheren Ergebnissen im konventionellen SYNTAX-Score der Fall.

\section{Fazit}

Mit dem individualisierten SYNTAXScore II konnten innerhalb einer Risikogruppe Patienten mit einer abweichenden Überlebenswahrscheinlichkeit identifiziert werden. Er sei eine aussagekräftigere Entscheidungshilfe als der ursprüngliche SYNTAX-Score vor einer perkutanen oder operativen Intervention, so die Autoren.

Sponsoring: Die Studie wurde von Boston Scientific finanziert.

Dr. med. Susanne Krome, Melle

DOI 10.1055/s-0033-1338011 